\title{
Universiteit
}

Leiden

The Netherlands

\section{Third trimester screening for alloimmunisation in Rhc-negative pregnant women: evaluation of the Dutch national screening programme}

Slootweg, Y.M.; Koelewijn, J.M.; Kamp, I.L. van; Bom, J.G. van der; Oepkes, D.; Haas, M. de

\section{Citation}

Slootweg, Y. M., Koelewijn, J. M., Kamp, I. L. van, Bom, J. G. van der, Oepkes, D., \& Haas, M. de. (2016). Third trimester screening for alloimmunisation in Rhc-negative pregnant women: evaluation of the Dutch national screening programme. Bjog: An International Journal of Obstetrics And Gynaecology, 123(6), 955-963. doi:10.1111/1471-0528.13816

Version: $\quad$ Not Applicable (or Unknown)

License: $\quad$ Leiden University Non-exclusive license

Downloaded from: https://hdl.handle.net/1887/113137

Note: To cite this publication please use the final published version (if applicable). 


\section{Third trimester screening for} alloimmunisation in Rhc-negative pregnant women: evaluation of the

\section{Dutch national screening programme}

Slootweg, Yolentha $M{ }^{1}$ Koelewijn, Joke $M,{ }^{2,3,4}$ van Kamp, Inge $L^{1}{ }^{1}$ van der Bom, Johanna $G^{5}$, Oepkes, Dick ${ }^{1}$, de Haas, Masja ${ }^{3}$

\footnotetext{
${ }^{1}$ Department of Obstetrics, Leiden University Medical Centre, Leiden, the Netherlands.

${ }^{2}$ Department of Obstetrics and Gynaecology, Academic Medical Centre, University of Amsterdam, Amsterdam, the Netherlands.

${ }^{3}$ Department of Experimental Immunohaematology, Sanquin Research, Amsterdam, the Netherlands.

${ }^{4}$ Department of General Practice, University Medical Center, Groningen

${ }^{5}$ Center for Clinical Transfusion Research, Sanquin Research \& Department of Clinical Epidemiology, Leiden University Medical Center, Leiden, The Netherlands Correspondence: Ms. Yolentha M Slootweg, Leiden University Medical Center, Dept of Obstetrics, PO Box 9600, 2300 RC Leiden, The Netherlands. Email: y.m.slootweg@lumc.nl
} 


\section{Abstract}

31 Objective: To evaluate the effect of red blood cell (RBC) antibody screening in the $27^{\text {th }}$ week of

32 pregnancy in Rhc-negative women, on detection of alloimmunisation, undetected at first

33 trimester screening ('late' alloimmunisation), and subsequent Haemolytic Disease of the Fetus

34 and Newborn (HDFN);to assess risk factors for late alloimmunisation.

35 Design: Prospective cohort and nested case-control study.

36 Setting: The Netherlands.

37 Population: Two-year nationwide cohort.

38 Methods: Prospectively inclusion of Rhc-negative women with negative first trimester screening

39 and of screen-negative controls.

40 Main outcomes measures: Late alloimmunisation, HDFN.

41 Analysis: Assessment of incidence and Numbers Needed to Screen (NNS) of late

42 alloimmunisation and HDFN; logistic regression analysis to establish risk factors for late

43 alloimmunisation.

44 Results: Late alloimmunisation occurred in 99/62,096 (0.159\%) of Rhc-negative women, 90\%

45 had c-/E-antibodies, 10\% non-Rhesus-antibodies. Severe HDFN (foetal/neonatal transfusion)

46 occurred in 2/62,096 (0.003\%) of Rhc-negative women and $2 \%$ of late alloimmunisations;

47 moderate HDFN (phototherapy) occurred in 20 children (22.5\%;95\%-Cl:13.8-31.1\%). Perinatal

48 survival was $100 \%$. The NNS to detect one HDFN case was $2,823(31,048$ for severe, 3,105 for

49 moderate HDFN). Significant risk factors were former blood transfusion OR 10.4;95\%-Cl:1.14-

50 94.9), parity (P-1 OR 11.8;95\%-Cl:3.00-46.5;P:>1 OR 7.77;95\%-Cl:1.70-35.4) and

51 amniocentesis/chorionic villus sampling during current pregnancy (OR 9.20;95\%-Cl:1.16-72.9).

52 Conclusion: Additional screening of Rhc-negative women improved detection of late

53 alloimmunisation and HDFN, facilitating timely treatment, with a NNS of 2,823. Independent risk 
54 factors for late alloimmunisation were blood transfusion, parity and chorionic villus

55 sampling/amniocentesis in the current pregnancy. The occurrence of most factors before the

56 current pregnancy suggests a secondary immune response explaining most late

57 alloimmunisations.

58 Tweetable abstract: 3rd trimester screening for alloimmunisation in Rhc-neg women improves

59 detection and treatment of severe HDFN.

60 Keywords: alloimmunization, screening, Rhc-negative, risk factors, incidences.

61

62 


\section{Introduction}

64 Haemolytic Disease of the Fetus and Newborn (HDFN) is caused by maternal alloimmunisation

65 against paternally inherited fetal red blood cell (RBC) antigens. HDFN may lead to fetal anaemia,

66 hydrops, asphyxia, perinatal death, and neonatal hyperbilirubinaemia, that may cause

67 'kernicterus'. Kernicterus can result in neurodevelopmental impairment with athetoid cerebral

68 palsy, hearing problems and psychomotor handicaps. ${ }^{1-7}$ Most severe HDFN cases are caused by

69 RhD-, Rhc- and Kell-antibodies (hereafter called anti-D, anti-c, etcetera). ${ }^{1-5,8}$ Timely detection of

70 maternal alloimmunisation facilitates fetal monitoring, aimed to identify fetuses with severe

71 disease needing intrauterine transfusions (IUT) and/or preterm delivery followed by

72 phototherapy or (exchange) transfusions. These therapies have all contributed to a considerable

73 decrease in HDFN-related perinatal death and long-term sequelae. 9, 10

74 Most Western countries have maternal alloimmunisation screening programmes. A wide

75 variation in design of these programmes exists between and within countries, ranging from

76 several screenings in all pregnant women to a single screening of RhD-negative women only. ${ }^{111-}$

$77 \quad 15$

78 In the Netherlands, all pregnant women are screened for RBC antibodies at the booking visit;

79 screening is repeated in week 27 for RhD-negative women, and since July 2011 also for Rhc-

80 negative women. ${ }^{16,17}$ Implementation of screening in Rhc-negative women, comprising $18.7 \%$

81 of pregnancies ${ }^{18}$, was based on a nationwide study in 400,000 pregnancies, showing that $25 \%$ of

82 severe HDFN cases in RhD-positive women occurred unexpectedly, after a negative screening

83 result in the first trimester. Some of these unexpected cases suffered from HDFN-related

84 handicaps due to perinatal asphyxia or kernicterus, because fetal anaemia and

85 hyperbilirubinaemia were not timely detected. In contrast, all cases of alloimmunisation 
detected at first trimester screening were timely treated and children were healthy at the age of

87 one year. ${ }^{8}$ All first trimester screen-negative cases of severe HDFN were caused by anti-c and/or

88 anti-E. However, long-term sequelae were only found in anti-c cases. ${ }^{8}$ Based on this outcome an

89 additional screening of all Rhc-negative women in week 27 was set-up to increase the detection

90 rate of severe HDFN cases with $25 \%$ (from 75 to 100\%). Undetected, these cases might result in

91 severe anaemia, hydrops, death or (too) late treatment of icterus.

92 So far, a few smaller studies showed no advantage of a second screening in RhD-positive

93 women. ${ }^{19-23}$ In the current large nationwide study, we set out to assess the incidence of HDFN

94 after a positive antibody screening in week 27 in Rhc-negative pregnant women and evaluated

95 whether implementation of this third trimester screening improved timely diagnosis and

96 treatment of HDFN. In addition, we aimed to identify risk factors for alloimmunisation first

97 recognized late in pregnancy, in order to provide insight in the causative mechanism in order to

98 be able to develop strategies for the prevention and timely detection of late alloimmunisation.

\section{Methods}

\section{Setting and Prevention programme in the Netherlands}

101 In the Netherlands, all pregnant women are typed for $\mathrm{ABO}, \mathrm{RhD}$ and Rhc blood group antigens

102 and screened for RBC antibodies at the first trimester booking visit. All RhD- and Rhc-negative

103 women, without RBC antibodies at the initial screening, are screened again in week $27 .{ }^{17}$ This

104 repeated screening is centralised in the laboratory of Sanquin Diagnostics in Amsterdam. When

105 clinically relevant RBC antibodies are detected, i.e. antibodies with the potency to destroy fetal

106 RBC's, the antibody titre and the Antibody Dependent Cellular Cytotoxicity Test (ADCC) are

107 performed, in order to assess the ability of these antibodies to cause fetal haemolysis. The 
108 father of the fetus is typed for cognate antigen(s) and in case of heterozygosity, non-invasive 109 typing on fetal DNA in maternal plasma is offered (for $R H D, R H C, R H c, R H E$ and $K$ )..$^{24}$ If the fetus 110 does not have the cognate antigen(s), further monitoring of the pregnancy is not necessary. If

111 the fetus is diagnosed as antigen-positive, the pregnancy is frequently monitored by laboratory 112 testing. In the presence of non-RhD RBC antibodies, an antibody titre $\geq 1: 16$ and/or ADCC test $113 \geq 30 \%$ indicates a major risk for HDFN, and fetal anaemia is monitored with middle cerebral

114 artery (MCA) Doppler measurements. ${ }^{25,}{ }^{26}$ Severe fetal anaemia is treated with intrauterine 115 transfusion(s) (IUT's) at the Leiden University Medical Centre (LUMC), which is the national 116 Dutch referral centre for management and treatment of pregnancies complicated by maternal 117 red cell alloimmunisation. In the Netherlands this study design does not require formal approval 118 of the Medical Ethical Committee.

\section{Study design}

120 To assess the occurrence of HDFN in Rhc-negative women diagnosed with newly detected RBC 121 antibodies (cases) at week 27 of pregnancy ('late alloimmunisation'), we prospectively collected 122 data on all these women and their offspring in the Netherlands between October $1^{\text {st }} 2011$ and 123 October $1^{\text {st }} 2013$.

125 The association between potential risk factors for late alloimmunisation and the occurrence of 126 late alloimmunisation among Rhc-negative pregnant women was examined in a case-control 127 study comprising Rhc-negative women with (the cases) and without (the controls) late 128 alloimmunisation, sampled between October $1^{\text {st }} 2011$ and October $1^{\text {st }} 2012$. Our planned study 129 period was one year. To obtain a more reliable estimation of the incidence of severe HDFN we 130 extended the study period with one year. We did not prolong the case-control study. 
131 Cases and controls were identified at Sanquin Diagnostics Amsterdam. For each case, three

132 controls were selected. These were the first three Rhc-negative women that were screened

133 negative, directly following the alloimmunised Rhc-negative woman.

\section{Outcomes}

135 The primary outcome was the incidence of severe and moderate HDFN in the offspring of Rhc-

136 negative pregnant women with antibodies first detected at 27 weeks gestation. Severe HDFN

137 was defined as alloimmune disease with the need for intrauterine transfusion and/or neonatal

138 exchange or blood transfusions in the first week of life. Moderate HDFN was defined as the need

139 for treatment of neonatal jaundice with phototherapy only. Long-term sequelae are all long

140 term impairments, most likely associated with the severe HDFN, such as kernicterus and/or

141 perinatal asphyxia.

142 Potential risk factors

143 We hypothesized that late in pregnancy detected alloimmunisations may emerge from a

144 primary immune response during the current pregnancy or from a secondary immune response,

145 triggered by fetomaternal (micro-)transfusions (FMT) of antigen-positive RBCs. ${ }^{12,}{ }^{20}$ Data on

146 known risk factors for red cell alloimmunisation, including risk factors for FMT during the

147 current pregnancy were collected in cases and controls.

\section{Data collection}

149 For inclusion of cases and controls, two of the researchers (YS, JK) contacted the obstetric care

150 provider (midwife, general practitioner and/or obstetrician) to explain our study. The obstetric

151 care provider asked the pregnant woman for consent for data collection and collection of cord

152 blood, to be sent to our laboratory by post. 
153 During the first year of the study, data on potential risk factors were collected during pregnancy,

154 immediately after consent was given, from the obstetric care provider and/or from the pregnant

155 woman. Potential risk factors comprised both general risk factors and in-pregnancy risk factors.

156 General risk factors included factors of general history (RBC transfusions, surgery,

157 haematological diseases), as well as gravidity and parity. 'In-pregnancy risk factors' were factors

158 within the previous pregnancy (gender child, caesarean section, surgical removal of placenta

159 and postpartum haemorrhage $(>1 L)$, and factors during the current pregnancy until week 27

160 (vaginal bleeding, abdominal trauma and invasive diagnostic and therapeutic interventions). ${ }^{27-30}$

161 To assess the occurrence of mild or severe HDFN in the study group, we collected the results of

162 laboratory monitoring during pregnancy from Sanquin Diagnostics, data of clinical monitoring

163 and IUT treatment during pregnancy, if applicable, from the LUMC, and neonatal outcome data

164 about treatment with blood transfusion(s) or phototherapy from the obstetric care provider,

165 from the paediatrician, from hospital laboratories and/or from the mothers, within two months

166 after birth.

167 All data were collected by questionnaires, which were completed by phone, e-mail or by post.

\section{Data analyses}

170 We assessed the incidence of late alloimmunisation as proportion of all screened Rhc-negative

171 women at 27 week of gestation and the occurrence of severe and moderate HDFN in association

172 with late immunisation. The cases with HDFN were classified by antibody specificity. When

173 multiple antibodies were present, the antibody specificity for which the paternal antigen was

174 positive and/or with the highest estimated risk for development of HDFN was considered as

175 'dominant' antibody. 
176 We calculated the Number Needed to Screen (NNS) to detect one case with severe HDFN timely,

177 assuming that none of these cases would have been detected without the third trimester

178 screening programme in Rhc-negative women. We also calculated the NNS to detect one case

179 with moderate HDFN and to detect one case of 'late alloimmunisation'. The NNS were

180 calculated as 1/(0-incidence of severe/moderate HDFN/late alloimmunisation in Rhc-negative

181 women, screened in the third trimester).

182 Dichotomous outcomes were described as number and percentage, normally distributed

183 continuous variables as mean and standard deviation and not-normally distributed continuous

184 variables as median and range.

185 The association between potential risk factors and the occurrence of late alloimmunisation was

186 examined with logistic regression, firstly by univariate and secondly by multivariate analysis.

187 Potential 'general' risk factors and in-pregnancy risk factors during the current pregnancy were

188 included in the first logistic model. Potential in-pregnancy risk factors originating from the

189 previous pregnancy were included in a second logistic model. Interactions between the

190 covariates were tested formally. All statistical analyses were performed with the Statistical

191 Package for the Social Sciences (SPSS) 21.0.

\section{RESULTS}

\section{Study population and response}

194 From October $1^{\text {st }} 2011$ till October $1^{\text {st }} 2013,62,096$ Rhc-negative women, without RBC

195 antibodies in the first trimester of pregnancy, were screened again in week 27 of gestation. Of

196 these, $99(0.16 \% ; 95-\mathrm{Cl} 0.13-0.19 \%)$ had newly detected clinically relevant RBC antibodies

197 (Figure 1). During the first year of the study, 168 controls were selected (matched to 54 cases), 
198 of which 104 (62\%) gave consent to collect data. The proportions of nulliparae, primiparae and

199 multiparae in the control group were $47.1 \%$ (95\%-Cl 34.1-60.1\%), 35.6\% (95\%-Cl 24.3-46.9\%)

200 and $18.5 \%(95 \%-\mathrm{Cl}: 2.7-34.3 \%)$ respectively, compared to proportions of $44.9 \%, 35.9 \%$ and

201 19.2\% respectively in the Netherlands in $2012 .^{31}$

202 From the newly immunised pregnant women, 10\% (10/99) refused participation in the study.

203 None of these women had either titres or ADCC values above the cut-off to select high-risk

204 cases, or was referred to the LUMC, the national referral centre for severe alloimmunised

205 pregnancies. Therefore, the occurrence of severe fetal haemolytic disease in the non-consent

206 group is very unlikely, although severe neonatal HDFN cannot be completely ruled out.

207 Therefore, incidences for severe HDFN are described in the whole group, but for moderate

208 HDFN only in the group with consent.

210 Incidence of late alloimmunisation

211 From the 99 late alloimmunisations, anti-c was the most frequently detected alloantibody

$212(65 / 99 ; 66 \%)$, in 20 cases anti-c was present in combination with anti-E and in seven cases with

213 other antibodies. Anti-E was present in 45/99 (45\%) cases, in 25 as a single antibody specificity.

214 In 54 cases with anti-c and 36 with anti-E the father was tested for the cognate antigen(s) and

215 was found to be positive in 53 and 35 cases, respectively. For the remaining 17 antibody

216 specificities, the father was typed in 14 cases and appeared positive for the cognate antigen(s)

217 in 5 cases (Table 1). The NNS to detect one late alloimmunisation was 628 (Table 2).

\section{Incidence of HDFN}

219 Severe HDFN due to RBC antibodies first detected at 27 weeks, occurred in two of the 62,096

220 Rhc-negative pregnancies screened and $2.0 \%$ of screen positive pregnancies (Table 2). One 
221 severe case was caused by the combination of anti-c and anti-E, mostly by anti-E (titre 1:256).

222 During this pregnancy, one IUT (pre-transfusion $\mathrm{Hb} 9.0 \mathrm{~g} / \mathrm{dL}$ ) was performed at 30+3 weeks,

223 followed by induction of labour at 36 weeks. The $\mathrm{Hb}$ and $\mathrm{Ht}$ levels postpartum were $12.4(\mathrm{~g} / \mathrm{dL})$

224 and 0.42 , respectively. Phototherapy was given during seven days. An exchange transfusion was

225 needed after two operations for pyloric stenosis, carried out after the first week of life. Two

226 months postpartum this child was confirmed to be in a good condition. The other severe case

227 was caused by anti-c only. No intrauterine transfusion was given. Labour was induced at 36

228 weeks +4 days; $\mathrm{Hb}$ and $\mathrm{Ht}$ at birth were $13.3(\mathrm{~g} / \mathrm{dL})$ and 0.42 , respectively. The lowest $\mathrm{Hb}$ was

$2299.8(\mathrm{~g} / \mathrm{dL})$, five top-up transfusions were given, no exchange transfusions were needed.

230 Phototherapy was given in 20 cases ( 12 anti-c, 5 anti-E and 3 anti-c and anti-E), resulting in an

231 incidence of moderate HDFN of $0.032 \%$ of all screened Rhc-negative women (Table 2 ) and

$23220.20 \%$ of screen-positive pregnancies. In cases with known outcome $(n=89)$ the incidence of

233 moderate HDFN was 22.5\%(95\%-Cl:13.8-31.1\%).

234 The NNS to detect one case of severe HDFN was 31,048 and to detect one case of moderate

235 HDFN 3,105.

236 Six cases of moderate HDFN occurred in association with laboratory test results below the

237 aforementioned cut-offs.

238 Forty-nine children of the 90 pregnancies with anti-c and/or anti-E, were antigen-positive for the 239 cognate antigens (based on antigen typing of the child $(n=26)$ or homozygosity of the father for

240 the antigens concerned $(n=23))$, five were antigen-negative and in 36 cases the antigen-typing

241 was unknown. We calculated that 17 children with unknown antigen-typing should have been

242 antigen-positive (Box S1), resulting in a risk for moderate HDFN in antigen-positive

243 fetuses/children from c-/E-immunised pregnancies of $30.35 \%(20 / 66 ; 95 \%-\mathrm{Cl} 24.6-36.0 \%)$. 


\section{Interventions for maternal alloimmunisation}

245 Preterm induction of labour was performed in both severe cases. In addition, 13 term inductions

246 were performed at least in part based on the presence of RBC antibodies (Figure S1), without

247 signs of fetal anaemia on ultrasound or Doppler. Five of the six cases with antibody titres and/or

248 ADCC test results above the cut-off values used in the Netherlands to indicate high-risk cases

249 needed phototherapy treatment. None of the seven cases of induced labour, with laboratory

250 testing results below the cut-offs, needed treatment for HDFN. Two of the phototherapy cases

251 were born prematurely (gestational age 28 and 34 weeks respectively), which was not

252 associated with the maternal alloimmunisation. Twenty-four children were admitted to the

253 neonatal ward, of which 20 were treated with phototherapy only. This concerned almost one

254 third of anti-c cases, $14 \%$ of only anti-E cases, and none of the cases with other antibodies.

\section{Risk Factors for late alloimmunisation}

256 A history of RBC transfusion, major surgery, previous parity, maternal age were, as well as

257 amniocentesis/chorion villus sampling in the current pregnancy were univariately associated

258 with the occurrence of late alloimmunisation in Rhc-negative women (Table S1).

259 Potential risk factors within previous pregnancies were not associated with late

260 alloimmunisation.

261 RBC transfusion, parity and amniocentesis/chorion villus sampling in the current pregnancy

262 were statistically significant independent risk factors for late alloimmunisation (Table 3). 


\section{Discussion}

\section{Main findings}

265 Late alloimmunisation, detected at $27^{\text {th }}$ week screening, occurred in $0.16 \%$ of all pregnancies of 266 Rhc-negative women. Within the group of late alloimmunisation, the risk for severe HDFN was $2672 \%$ and for moderate HDFN 22.5\%. Most new immunisations and all HDFN cases were caused 268 by anti-c and/or anti-E. Amniocentesis or chorionic villus sampling in the current pregnancy, as 269 well as parity and a history of RBC transfusion were independent risk factors for 270 alloimmunisation detected late in pregnancy.

\section{Strengths and limitations}

272 To our knowledge this is the first prospective nationwide study on the effect of a second

273 antibody screening in Rhc-negative women. Our study provides a reliable estimation of the

274 incidence of late alloimmunisation and subsequent HDFN. Although outcome data of $10 \%$ of the 275 cases were missing, severe HDFN is very unlikely in these cases, because laboratory results were 276 not above the cut-off values indicating high-risk for HDFN and no cases needed monitoring in

277 the national referral centre. Moreover, in some cases it was impossible to separate the

278 contribution of alloimmunisation from other causes for hyperbilirubinaemia, for example in two

279 prematurely born children. This may have caused an -at most slight- overestimation of the 280 incidence of moderate HDFN.

282 One third of the controls did not participate in our study, which may have caused selection bias 283 in our risk factor analysis. Most common reasons for non-participating were a language barrier, 284 social problems and declined cooperation of the obstetric caregiver, reasons unlikely associated 
285 with risk factors for alloimmunisation. This was supported by the distribution of parity, a strong

286 risk factor, in our control group, which did not differ from national data.

287 Some risk factors showed wide confidence intervals, due mainly to limited numbers. We

288 consider it unlikely that with increased numbers and thus narrowed confidence intervals,

289 the risk estimations would turn out different.

\section{Previous findings and interpretation}

291 The incidence of late alloimmunisation in Rhc-negative women was in line with expectations

292 following our former evaluation of the Dutch screening programme for non-RhD antibodies. ${ }^{8}$ No

293 studies are available yet in which only Rhc-negative women were screened for late

294 alloimmunisation. A small Dutch study in which RhD-positive women underwent a second

295 screening reported higher incidences of late alloimmunisation, which might at least partly be

296 explained by the fact that this study was performed in a population of parous women, at

297 increased risk for alloimmunisation. ${ }^{32}$ Studies including 3,000-70,000 RhD-positive pregnant

298 women reported incidences of late alloimmunisation varying between 0.06 and $0.43 \%$, in line

299 with our data. ${ }^{33}$ The incidence of late alloimmunisation in Rhc-negative women might be

300 somewhat higher than in all RhD-positive women, since anti-c and anti-E, the most frequent

301 newly detected antibodies in all studies, are found especially in Rhc-negative women.

302 Remarkably, the incidence of severe HDFN in cases with late alloimmunisation was considerably

303 lower than expected, resulting in a NNS to detect one severe HDFN case of 31,048 . Based on the

$3040.002 \%$ incidence of severe HDFN by late alloimmunisation, found in our study in $2003-2004{ }^{8}$ a

305 NNS of about 9,000 was expected. An explanation for this decreased incidence might be that

306 timely detection of cases at risk for fetal haemolysis, followed by labour induction in week 37 , as

307 advised in the Dutch Guideline on maternal alloimmunisation, preventing the development to 
308 severe HDFN in some cases. ${ }^{34}$ This explanation is supported by the shorter median gestational

309 age in cases with labour induction, followed by phototherapy treatment, than in the missed

310 severe HDFN cases in our former study (265 versus 274 days). Moreover, the increased

311 availability of intensive phototherapy combined with the introduction of a new guideline in 2008

312 including a more conservative approach concerning the use of exchange transfusions to lower

313 bilirubin levels, will have reduced the use of exchange transfusions.

314 Both severe cases of HDFN in our study, were probably not detected without the screening

315 programme. These were uncomplicated pregnancies and normally developed fetuses. Current

316 standard of care for such pregnancies in The Netherlands does not include routine ultrasound in

317 the third trimester. Even if ultrasound would be done, without a high index of suspicion specific

318 anaemia detection by middle cerebral artery Doppler would not have taken place. Clinically,

319 only reduced fetal movements and hydrops on ultrasound would be detected, which are very

320 late stages of disease associated with a significant perinatal death risk. Therefore, we

321 hypothesize that the remarkable decrease of the incidence of severe HDFN by late

322 alloimmunisation, for which no other explanation can be given, is a benefit of the

323 implementation of third trimester screening in Rhc-negative women, a benefit that highly

324 exceeds the benefit as suggested by the NNS of 31,048 .

325

326 A possible negative feature of screening might be a number of relatively early inductions of

327 labour because of maternal alloimmunisation, despite laboratory test results being below the

328 cut-offs, as was the case in $50 \%$ of term inductions. It should be kept in mind that in these cases,

329 factors other than maternal alloimmunisation may have contributed to the decision to induce

330 labour. It was however reassuring that the induction rate in cases was comparable with national

331 figures (17.2 versus $21.4 \%)^{31}$ 
333 One severe HDFN case occurred in a pregnancy complicated by low anti-c and high anti-E levels,

334 while three moderate cases were due to anti-E only. This raises the question whether also

335 women with an Rhc-positive but RhE-negative phenotype (CcDee (35\%) or ccDee $(1,6 \%)^{20}$ should

336 be offered a second screening. Our former evaluation showed only one missed case during two

337 years with the CcDee phenotype, while all cases with long term sequelae were caused by anti-c. ${ }^{8}$

338 Therefore, expanding the screening to all RhE-negative women will most likely not significantly

339 improve the detection of severe HDFN cases. Registration of screen-undetected cases with

340 HDFN would be helpful to clarify this issue.

341

342 We identified risk factors before as well as during the current pregnancy. Parity and blood

343 transfusion were identified in our former study as risk factors for early alloimmunisation. ${ }^{21}$

344 These findings are in accordance with the hypothesis that the primary immune response

345 occurred already in, or following, a previous pregnancy. Antibody levels then fall too low to be

346 detected at first trimester screening, and rise again after renewed contact during pregnancy of

347 the maternal immune system with fetal red cells. This might have occurred after amniocentesis

348 or chorionic villus sampling, when these cases also had one or more risk factors before the

349 current pregnancy. The contribution of each of the risk factors is difficult to be estimated in this

350 relatively small study. In the risk factor analysis only cases from the first year of the study with

351 consent to collect data on risk factors $(n=46)$ were included. We did not match for potential

352 confounders, because, as described by Altman (1991), any variable used for matching cannot be

353 investigated as a possible risk factor for maternal alloimmunisation. ${ }^{35}$ As this is the first study on

354 risk factors for late alloimmunisation, we aimed to investigate all possible risk factors instead of 
355 collecting variables, known as risk factors for maternal alloimmunization detected at first

356 trimester screening only.

357 Our analysis underlines a restrictive blood transfusion policy, as well as the use of Rhc- and RhE-

358 matched donor blood, according to current Dutch guidelines. ${ }^{36}$ Moreover, invasive diagnostic

359 procedures are associated with fetomaternal haemorhage ${ }^{29}$, which can cause a primary or

360 secondary immune response, the latter with a rapid rise of maternal RBC antibody levels. This

361 underlines the importance of non-invasive prenatal testing (NIPT). ${ }^{37}$

362 Theoretically, third trimester screening in Rhc-negative women may be restricted to women

363 with risk factors, $62 \%$ of the pregnant women in our control group. However, subgroup first

364 trimester screening, as advised by the Dutch Health Council ${ }^{16}$, was not implemented, because of

365 practical objections of the obstetric care workers. Our study confirms the usefulness of the

366 additional third trimester screening for RBC alloantibodies in all Rhc-negative women.

367 Our previously published economic analysis showed that the extra costs of the expanded

368 screening programme in the Netherlands are about 1.4 M€/year. As we detected two severe

369 cases during two years, this means 1.4 M€/case, which is lower than the estimated life time

370 costs of a surviving child with long term sequelae, which are about $3 \mathrm{M}$ euro, when this person

371 reaches the age of 60 years. ${ }^{38}$ We also showed that the psychological burden of antibody

372 screening is small and balanced with the benefits. ${ }^{39}$

\section{Conclusion}

374 A repeated RBC antibody screening in week 27 of pregnancy in Rhc-negative women contributes

375 to the timely detection and treatment of severe HDFN and most likely also leads to a decrease

376 of the incidence of severe HDFN. An optimal management eventually results in less severely

377 compromised cases and a reduction in the long-term morbidity and mortality associated with

378 severe HDFN. 


\section{Acknowledgements}

380 We thank all the pregnant women and obstetric care providers who participated in the study.

381 Cases and controls were identified at Sanquin Diagnostics Amsterdam (Dr. C. Folman and Ms. H.

382 Woortmeijer are acknowledged for making data of their laboratory registries available for the 383 study).

\section{Disclosure of interests}

451 There are no competing interests to declare. The ICMJE disclosure forms are available as online 452 supporting information.

\section{Contribution of authorship}

454 YM Slootweg designed the study, carried out data collection, extraction, analysis and

455 interpretation of data and drafted the article and is responsible for the integrity of the work as a 456 whole. JM Koelewijn advised on study design, carried out data collection, extraction and

457 interpretation of data, revised the article critically for intellectual content and approved the final 458 draft for publication. M. de Haas advised on study design, carried out interpretation of the data, 459 revised the article critically for intellectual content, and approved the final draft for publication. 460 JG van der Bom, IL van Kamp and D Oepkes assisted with interpretation of the data, revised the 461 article critically for intellectual content and approved the final draft for publication.

\section{Ethics approval}

463 In the Netherlands this study design does not require formal approval of the Medical Ethical 464 Committee. 


\section{Funding}

466 This study was conducted in a partnership of Sanquin Diagnostics Amsterdam and Leiden

467 University Medical Centre. This study was not funded by external sources

\section{Reference List}

469

470

471

472

473

474

475

476

477

478

479

480

481

482

483

484

485

486

487

488

489

490

491

492

493

494

495

496

497

498

499

500

501

502

503

504

1. Daniels GL. Blood group antibodies in haemolytic disease of the fetus and newborn; in: Hadley A, Soothill P (eds): Alloimmune disorders of pregnancy. Anaemia, thrombocytopenia and neutropenia in the fetus and newborn. 2002.

2. Moise KJ. Fetal anemia due to non-Rhesus-D red-cell alloimmunization. Seminars in fetal and neonatal medicine; Perinatal Haematology; 2008. p. 207-14.

3. Moise KJ. Management of rhesus alloimmunization in pregnancy. Obstetrics \& Gynecology. 2008;112(1):164-76.

4. Franklin I. Prevention of rhesus haemolytic disease of the fetus and newborn. Lancet. 2009;373(9669).

5. Klein HG AD. Haemolytic disease of the fetus and the newborn. In: Klein HG, Anstee DJ, editors. Mollison's blood transfusion in clinical Medicine. 2012:499-548.

6. Watchko J, Tiribelli C. Bilirubin-induced neurologic damage--mechanisms and management approaches. N Engl J Med. 2013;369(21):2021-30.

7. Bhutani VK ZA, Blencowe H, Khanna R, Sgro M, Ebbesen F et al. Neonatal hyperbilirubinemia and Rhesus disease of the newborn: incidence and impairment estimates for 2010 at regional and global levels. Pediatr Res. 2013;74(1):86-100.

8. Koelewijn JM, Vrijkotte TGM, van der Schoot CE, Bonsel GJ, de Haas M. Effect of screening for red cell antibodies, other than anti-D, to detect hemolytic disease of the fetus and newborn: a population study in the Netherlands. Transfusion. 2008;48(5):94152.

9. Lindenburg IT, Smits-Wintjes VE, van Klink JM, Verduin E, van Kamp IL, Walther FJ, et al. Long-term neurodevelopmental outcome after intrauterine transfusion for hemolytic disease of the fetus/newborn: the LOTUS study. Am J Obstet Gynecol. 2012;206(2):1418.

10. Lindenburg IT, van Klink JM, Smits-Wintjens VE, Oepkes D, Lopriore E. Long-term neurodevelopmental and cardiovascular outcome after intrauterine transfusions for fetal anaemia: a review. Prenat Diagn. 2013;33(9):815-22.

11. American Congress of Obstetricians and Gynecologists. Management of alloimmunization during pregnancy. Obstetrics \& Gynecology. 2006;108(2):457-64.

12. Koelewijn JM. Detection and prevention of pregnancy immunisation, The OPZI study. Academic thesis. 2009(Chapter 1):9-31.

13. Canadian Blood Services. Clinical Guide to Transfusion. Canadian Blood Services, editor. 2007.

14. Gooch A PJ, Wray J, Qureshi H. Guideline for blood grouping and antibody testing in pregnancy. Transfus Med. 2007;17(4):252-62. 
15. Gottvall T, Filbey D. Alloimmunization in pregnancy during the years 1992 untill 2005 in the central west region of Sweden. Acta Obstetricia et Gynecologica Scandinavica: Informa Scandinavian; 2008. p. 843-8.

16. Health Counsil of the Netherlands. Pregnancy immunisation by red blood cells. Advise Health Council. The Hague; 2009.

17. RIVM National Institute Public Health and Environment. Prenatal screening infectious diseases and erythrocyte antibodies. the Hague, the Netherlands; 2014.

18. Reid ME, Lomas-Francis C, Olsson ML. The blood group antigen facts book. 3 ed. Oxford: Elsevier; 2012.

19. Lurie S EE, Piper I, Woliovitch I. Is antibody screening in Rh (D)-positive pregnant women necessary? Fetal Neonatal Med. 2003;14(6):404-6.

20. Adeniji AA, Fuller I, Dale T, Lindow SW. Should we continue screening rhesus D positive women for the development of atypical antibodies in late pregnancy? Journal of Maternal-Fetal and Neonatal Medicine: Informa Clin Med; 2007. p. 59-61.

21. Andersen AS, Praetorius L, Jorgensen HL, Lylloff K, Larsen KT. Prognostic value of screening for irregular antibodies late in pregnancy in rhesus positive women. Acta Obstetricia et Gynecologica Scandinavica: Informa Scandinavian; 2002. p. 407-.

22. Dajak S, Stefanovic V, Capkun V. Severe hemolytic disease of fetus and newborn caused by red blood cell antibodies undetected at first-trimester screening (CME). Transfusion. 2011;51(7):1380-8.

23. Bowell PJ AD, Entwistle CC. Blood group antibody screening tests during pregnancy. British journal of obstetrics and gynaecology. 1986;96(10):1038-43.

24. Scheffer PG vdSC, Page-Christiaens GC, de Haas M. Noninvasive fetal blood group genotyping of rhesus $D, C, E$ and of $K$ in alloimmunised pregnant women: evaluation of a 7-year clinical experience. BJOG. 2011;118(11):1340-8.

25. de Haas M, Koelewijn JM, Bilgin K, Vrijkotte TGM, van der Schoot CE, Bonsel GJ. Diagnostic performance of laboratory monitoring to predict severe haemolytic disease of the fetus and newborn in non-RhD-alloimmunised pregnancies. Detection and prevention of pregnancy immunisation, The OPZI-study, Academic Thesis. 2009 2009; Chapter 4:73-85.

26. Oepkes D, Seaward PG, Vandenbussche FP, Windrim R, Kingdom J, Beyene J. Doppler ultrasonography versus amniocentesis to predict fetal anemia. N Engl J Med. 2006;355(2):156-64.

27. Koelewijn JM, Vrijkotte TGM, de Haas M, van der Schoot CE, Bonsel GJ. Risk factors for the presence of non-rhesus $D$ red blood cell antibodies in pregnancy. BJOG: An International Journal of Obstetrics \& Gynaecology. 2009;116(5):655-64.

28. Sebring ES, Polesky HF. Fetomaternal hemorrhage: incidence, risk factors, time of occurrence and clinical effects. Transfusion. 1990;30(4):344-57.

29. Sikovanyecz J HE, Pasztor N, Kereszturi A, Szabo J, Pal A. Fetomaternal transfusion after amniocentesis and cordocentesis. Ir J Med Sci. 2011;180(3):697-701.

30. Oxford CM LJ. Trauma in Pregnancy. Clin Obstet Gynecol. 2009;52(4):611-29.

31. Dutch association for perinatal registration. Perinatal Care in the Netherlands 2012. table 1.1.1; 2013.

32. de Vrijer B H-LE, Oosterbaan HP. The incidence of irregular antibodies in pregnancy: a prospective study in the region of 's-Hertogenbosch. Ned Tijdschrift Geneeskd. 1999;143(50):2523-7. 
551 33. Heddle NM, Klama L, Frassetto R, O'Hoski P, Leaman B. A retrospective study to determine the risk of red cell alloimmunization and transfusion during pregnancy. Transfusion.1993;33(3):217-20.

34. NVOG Dutch Association Obstetrics and Gynaecology. Red blood cell immunisation and pregnancy. 2009.

35. Altman D. Practical Statistics for Medical Research. London: Chapman and Hall/CRC 1991. p. 94.

36. CBO Central Guidance Agency. Guideline Blood Transfusion. Utrecht, the Netherlands; 2011.

37. Health Council of the Netherlands. NIPT: Dynamics and Ethics of Prenatal Screening. the Hague: Health Council of the Netherlands. 2013.

38. Koelewijn JM. Economic analysis of the screening programme for red blood cell antibodies, other than RhD, in pregnancy.; 2009. p. 107-30.

39. Koelewijn JM, Vrijkotte TGM, de Haas M, van der Schoot CE, Bonsel GJ. Women's attitude towards prenatal screening for red blood cell antibodies, other than RhD. BMC Pregnancy and Childbirth. 2008;8(1):49. 
Figure 1: Flowchart of inclusions and exclusions of cases and controls.

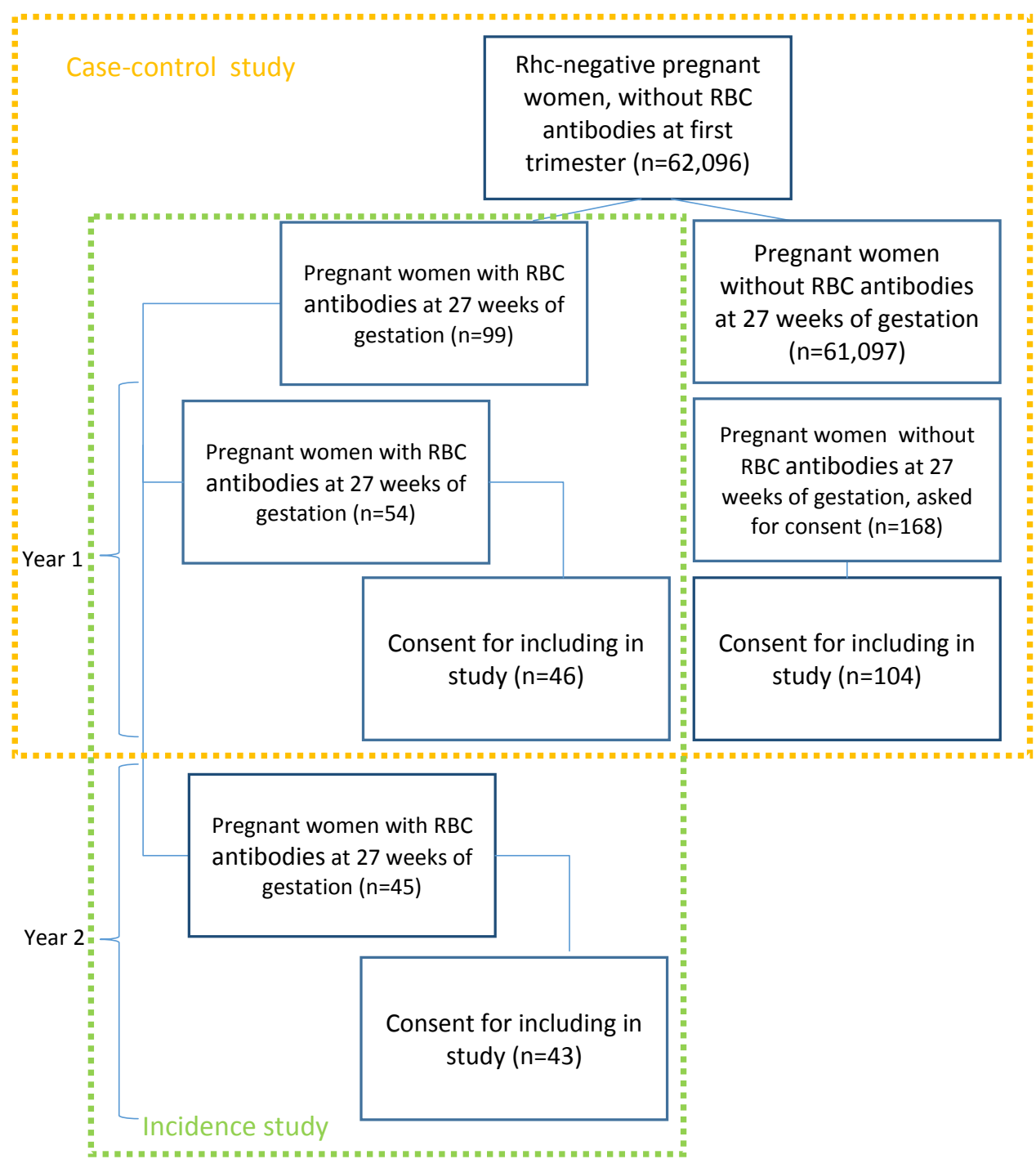


Table 1. Newly detected clinically relevant RBC antibodies in week 27 in Rhc-negative pregnant women

\begin{tabular}{|c|c|c|c|c|c|c|c|c|c|}
\hline \multicolumn{2}{|c|}{ Antibody specificity } & \multicolumn{2}{|c|}{$\mathrm{N}(\%)$} & \multicolumn{3}{|c|}{$\begin{array}{l}\text { Phenotype father antigen } \\
\text { dominant antibody* }\end{array}$} & \multirow{2}{*}{$\begin{array}{c}\text { Severe HDFN } \\
\text { IUT/(exchange) } \\
\text { transfusion }\end{array}$} & \multirow{2}{*}{$\begin{array}{c}\text { Moderate HDFN } \\
\text { Phototherapy only ** }\end{array}$} & \multirow[t]{2}{*}{$\begin{array}{l}\text { HDFN in lab } \\
\text { tests }>\text { cut-of }\end{array}$} \\
\hline $\begin{array}{l}\text { Dominant } \\
\text { antibody* }\end{array}$ & $\begin{array}{l}\text { Additional } \\
\text { antibodies }\end{array}$ & $N$ & $\%$ & negative & positive & ? & & & \\
\hline c & - & 38 & 38.4 & 1 & 30 & 7 & 1 & $9 / 34^{* * *}$ & $7 / 13$ \\
\hline $\mathrm{E}$ & - & 25 & 25.3 & 1 & 19 & 5 & 0 & $3 / 24$ & $2 / 3$ \\
\hline c & $\mathrm{E}$ & 14 & 14.1 & 0 & 11 & 3 & 0 & $2 / 12$ & $1 / 3$ \\
\hline E & c & 6 & 6.1 & 0 & 5 & 1 & 1 & $3 / 5$ & $3 / 3$ \\
\hline c & K & 1 & 1.0 & 0 & 1 & 0 & 0 & $1 / 1$ & $1 / 1$ \\
\hline c & $\mathrm{K}+\mathrm{Fy}^{\mathrm{a}}$ & 1 & 1.0 & 0 & 1 & 0 & 0 & $0 / 0$ & $0 / 0$ \\
\hline c & $\mathrm{Jk}^{\mathrm{a}}$ & 3 & 3.0 & 0 & 3 & 0 & 0 & $1 / 3$ & $1 / 1$ \\
\hline c & $\mathrm{Jk}^{\mathrm{b}}$ & 1 & 1.0 & 0 & 1 & 0 & 0 & $0 / 1$ & $0 / 0$ \\
\hline c & $W r^{a}$ & 1 & 1.0 & 0 & 1 & 0 & 0 & $1 / 1$ & $1 / 1$ \\
\hline K & - & 1 & 1.0 & 1 & 0 & 0 & 0 & $0 / 1$ & $0 / 0$ \\
\hline $\mathbf{J k}^{\mathrm{a}}$ & - & 2 & 2.0 & 0 & 2 & 0 & 0 & $0 / 2$ & $0 / 0$ \\
\hline s & - & 1 & 1.0 & 0 & 1 & 0 & 0 & $0 / 1$ & $0 / 0$ \\
\hline$c^{w}$ & - & 5 & 5.1 & 5 & 0 & 0 & 0 & $0 / 4$ & $0 / 0$ \\
\hline Total & & 99 & 100 & 8 & 75 & 16 & 2 & $20 / 89$ & $16 / 25$ \\
\hline
\end{tabular}

* Dominant antibody if multiple antibodies are present: antibody specificity for which the paternal antigen is positive and/or with the highest estimated risk for development of HDFN.

** Denominators for phototherapy: cases with known outcome.

*** In one antigen-positive child only a maximum bilirubin level of $\mathbf{2 8 9} \mu \mathrm{mol}$ was known, but data about phototherapy treatment were missing; this case was classified as moderate HDFN. 
Table 2. Calculation Numbers Needed to Screen (NNS) to detect late alloimmunisation in Rhc-negative women and subsequent disease.

\begin{tabular}{|c|c|c|c|c|}
\hline & \multicolumn{3}{|c|}{$\begin{array}{l}\text { Screened Rhc-negative women } 1 / 10 / 2011-1 / 10 / 2013 \\
\qquad N=62,096\end{array}$} & \multirow{2}{*}{$\begin{array}{c}\text { Numbers Needed to } \\
\text { Screen } \\
\text { to detect one case }\end{array}$} \\
\hline & n & $\begin{array}{c}\% \\
(95 \%-\mathrm{Cl}) \\
\text { of Rhc-negative women }\end{array}$ & $\begin{array}{c}\% \\
(95 \%-\mathrm{Cl}) \\
\text { of cases with late } \\
\text { alloimmunisation }\end{array}$ & \\
\hline $\begin{array}{l}\text { Late } \\
\text { alloimmunisation }\end{array}$ & 99 & $0.159(0.128-0.191)$ & & 628 \\
\hline HDFN & 22 & $0.035(0.021-0.050)$ & $22.22(12.94-31.51)$ & 2,823 \\
\hline - $\quad$ severe & 2 & $0.003(0-0.008)$ & $2.02(0-4.82)$ & 31,048 \\
\hline - moderate & 20 & $0.032(0.018-0.046)$ & $20.20(11.35-29.06)$ & 3,105 \\
\hline
\end{tabular}

Assumption calculation NNS: timely detection without screening programme $=0 \%$. NNS calculated as $1 /(0$ incidence in Rhc-negative women)

Formula for calculation of the $95 \%$-confidence intervals: $p-1.96 * R O O T(p *(1-p) / n)$, resp. $p+1.96 * R O O T(p *(1-$ $p) / n) \cdot p=$ proportion of alloimmunised women $(0.16 \%)$ and $n=$ the number of screened women $(62,096)$. 
Table 3. Associations between risk factors and late alloimmunisation

\begin{tabular}{|c|c|c|c|c|c|}
\hline & & $\begin{array}{l}\text { Cases } \\
\mathrm{N}(\%)\end{array}$ & $\begin{array}{l}\text { Controls } \\
\mathrm{N}(\%)\end{array}$ & Crude OR $(95 \%-\mathrm{Cl})$ & $\begin{array}{c}\text { Adjusted OR* * } \\
(95 \%-\mathrm{Cl})\end{array}$ \\
\hline General risk factors: & & $N=46^{*}$ & $\mathrm{~N}=104$ & & \\
\hline \multirow[t]{4}{*}{ Age } & $25-29$ & $8(17)$ & $33(32)$ & Ref & Ref \\
\hline & $<25$ & $4(9)$ & $15(14)$ & $1.10(0.29-4.23)$ & $1.38(0.27-6.99)$ \\
\hline & $30-34$ & 18 (39) & $37(36)$ & $1.90(0.72-4.96)$ & $1.21(0.39-3.71)$ \\
\hline & $>=35$ & $16(35)$ & $19(18)$ & $3.47(1.25-9.63)$ & $1.78(0.54-5.83)$ \\
\hline \multirow[t]{3}{*}{ Parity } & 0 & $3(7)$ & $49(47)$ & Ref & Ref \\
\hline & 1 & $30(65)$ & $37(36)$ & $13.2(3.75-46.7)$ & $11.81(3.00-46.5)$ \\
\hline & $>2$ & $13(28)$ & $18(17)$ & $11.8(3.01-46.3)$ & $7.77(1.70-35.4)$ \\
\hline RBC transfusion & & $6(13)$ & $1(1)$ & $15.45(1.80-132.4)$ & 10.39 (1.14-94.9) \\
\hline Major Surgery & & $18(40)$ & $21(20)$ & $2.64(1.23-5.66)$ & $2.37(0.96-5.86)$ \\
\hline \multicolumn{6}{|l|}{$\begin{array}{c}\text { In-pregnancy risk factors in } \\
\text { current pregnancy: }\end{array}$} \\
\hline $\begin{array}{c}\text { Chorionic villus } \\
\text { sampling/amniocentesis }\end{array}$ & & $6(13)$ & $2(2)$ & 7.65 (1.48-39.5) & $9.20(1.16-72.9)$ \\
\hline
\end{tabular}

* Proportions determined in group with known data; missing data maximum 1.

** Adjusted for maternal age, parity, RBC transfusion, major surgery and chorionic villus sampling/amniocentesis

Goodness of fit tests showed no evidence of lack of fit $(p=0.90)$; explained variance $36.7 \%$ (Nagelkerke Chisquare) 